\title{
Efficiency and angular resolution calculations for a prototype multiple Compton scatter camera
}

\author{
N. Dogan * and D.K. Wehe \\ Department of Nuclear Engineering, Unu'ersity of Michigan, Ann Arbor, MI 48109, USA
}

(Received 27 December 1993)

Monte Carlo simulations were performed to optimize the layer thickness of a Compton camera which utilizes multiple Compton scatterings. The optimum layer thickness was found to be less than $0.5 \mathrm{~mm}$ for $150 \mathrm{keV}, 2 \mathrm{~mm}$ for $511 \mathrm{keV}$ gamma rays and approximately $5 \mathrm{~mm}$ for $1000 \mathrm{keV}$ gamma rays. The efficiencies of two multiple Compton scatter cameras which consist of a 20 layer stack of either $1 \mathrm{~mm}$ or $4 \mathrm{~mm}$ thıck Si strip detectors surrounded by a $2 \mathrm{~cm}$ thıck $\mathrm{CsI}(\mathrm{Tl})$ side counter are presented for $150-1000 \mathrm{keV}$ gamma rays. The expected angular resolution of the proposed design ( $1 \mathrm{~mm}$ thick, 20 layer $\mathrm{Si})$ is presented for different assumed noise levels over the energy range $150-1000 \mathrm{keV}$.

\section{Introduction}

A multiple Compton scatter camera which consists of a stack of thin, large-area, energy and position sensitive detectors was first proposed by Kamea and Hanada [1]. Their conceptual design of a multiple Compton camera had 50 layers of $0.5 \mathrm{~mm}$ thick silicon strip detectors in a stack with the layers separated by 3 $\mathrm{mm}$ and surrounded by a high efficiency annular detector. Because of the thinness of the layers, the energy range of their design is limited to $150-600 \mathrm{keV}$. Fig. 1 shows a schematic drawing of a multiple Compton scatter imaging camera. While such a camera is more complex than a single Compton camera, it offers the potential of highly efficient measurements of the direction, energy, and polarization of the incident gamma rays.

In this work, we present the calculations for optimizing the detector layer thickness for a multiple Compton scatter imaging camera over the energy range of $150-1000 \mathrm{keV}$. Si was chosen as a detector material because of its excellent energy resolution, high Compton to photoelectric absorption ratio, silicon's ability to operate at room temperature and the interesting developments in silicon detector technology [2]. Based on this thickness optimization, a proposed design for a multiple Compton scatter imaging camera is given. The efficiency and angular resolution of the sample design are then presented.

\footnotetext{
* Corresponding author, tel. +13139369221, fax +1313 9367859 .
}

\section{Optimization of layer thickness}

One of the important design parameters for a multiple Compton scatter camera is the thickness of the detector layers. Since the desired interaction mode is Compton scattering, the probability of a single Compton scattering followed by an immediate escape of the scattered gamma ray should be maximized, while minimizing the probabilities of multiple scatterings and photoelectric events within a layer. These constraints argue for a large number of very thin layers. These very thin layers also contribute to higher angular resolution of the camera resulting from a decreased uncertainty in the reaction positions. However, a serious concern is the range of the Compton recoil electrons in the thin layers. The thickness of any layer should be large enough to contain the recoil electrons. If the recoil electron has enough energy, it may escape from the layer without depositing its full energy and may travel into an adjacent layer and deposit energy. While this condition may be identifiable by signals originating in adjacent layers or by the event not satisfying a sequence reconstruction algorithm [3], this leads to a loss in efficiency of the camera for high gamma ray energies if the layers are made too thin.

To examine the role of detector thickness on camera efficiency, Monte Carlo simulations were performed to calculate the fraction of recorded events $f_{\mathrm{ce}}$, which correspond to a single Compton scattering and then escape of the scattered gamma ray from the layer, and to calculate the probability $P_{\mathrm{ec}}$ of events in which the recoil electron is captured within a layer, for var1- 


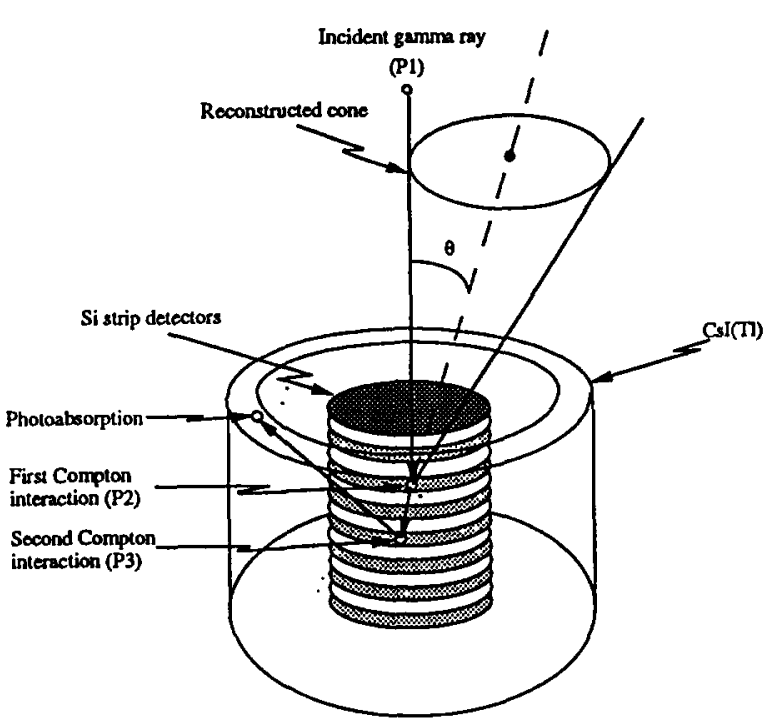

Fig. 1. Schematic drawing of a multiple Compton scatter imaging camera.

ous detector layer thicknesses. The expected number of measured events in a layer which correspond to a single Compton scattering with full recoil electron energy capture is proportional to $f_{\mathrm{ce}} P_{\mathrm{ec}}{ }^{\# 1}$. Figs. $2 \mathrm{a}-\mathrm{c}$ show the results for 150,511 and $1000 \mathrm{keV}$ incoming gamma rays for silicon detectors. As expected, $f_{\text {ce }}$ decreases while $P_{\mathrm{ec}}$ increases with increasing detector layer thickness, giving approximately $0.5,2$ and $5 \mathrm{~mm}$ optimum thicknesses for 150,511 and $1000 \mathrm{keV}$ gamma rays, respectively. The arrows show the maxima of $f_{\mathrm{ce}} P_{\mathrm{ec}}$ and are fairly broad. The results suggest that the layer thickness should be chosen thinner for low energies than for higher energies.

\section{Proposed camera design}

A multiple Compton scatter camera consisting of 20 layers of $1 \mathrm{~mm}$ thick Si strip detectors and surrounded by a $2 \mathrm{~cm}$ thick $\mathrm{CsI}(\mathrm{Tl})$ detector was simulated using the Integrated Tiger series of Monte Carlo Codes [4]. Each Monte Carlo simulation used a batch of 10000 gamma rays incident normally on the camera. The proposed design differs substantially from that of Kamea and Hanada in the relatively large $(\sim 1 \mathrm{~cm})$ layer spacing. The larger layer spacing is commensu-

\footnotetext{
\#1 Strictly speaking, thıs is true when these probabilities are independent. While there is some correlation between the probability of escape of the Compton recoll electron and the scattered photon, this correlation tends to average out over the many different incident directions and energies. This can be confirmed by the Monte Carlo results.
}

rate with the $1 \mathrm{~mm}$ strip detector pitch to ensure a small uncertainty in the cone axis. This layer spacing gives a $5^{\circ}$ angular uncertainty if two Compton scatterings occur in adjacent detector layers. Since the mean free paths of the gamma rays in $\mathrm{Si}$ are several centimeters in the gamma ray energy range $150-1000 \mathrm{keV}$, the average uncertainty in the cone axis orientation will be much smaller.

\subsection{Intrinsic efficiency}

Fig. 3 shows the intrinsic efficiency for the proposed design, where we define this efficiency to be the probability that an incident gamma ray will yield a sequence of measurable events which could yield a correct backprojection. Using the Monte Carlo generated data, the
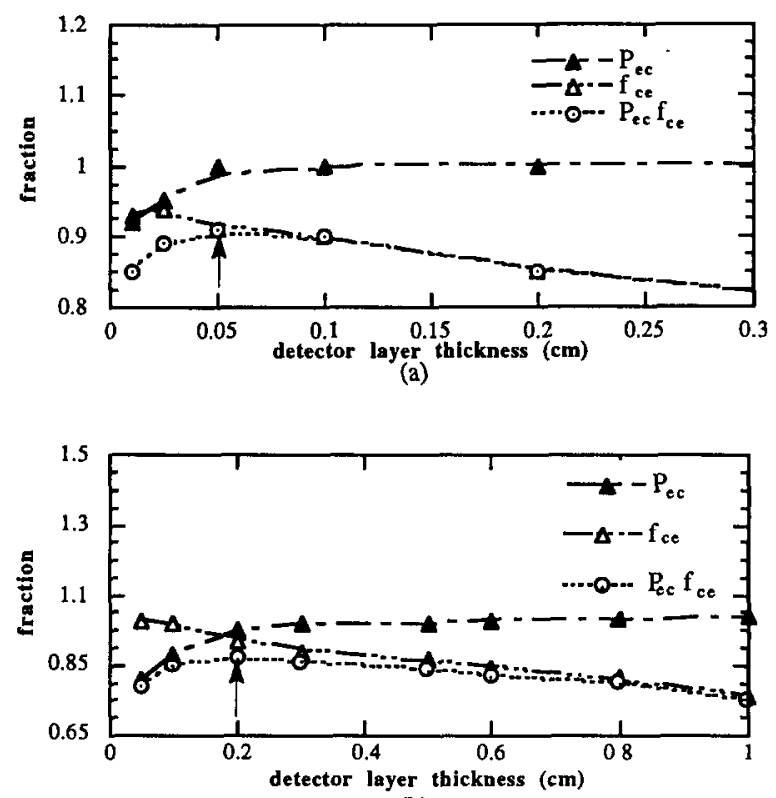

(b)

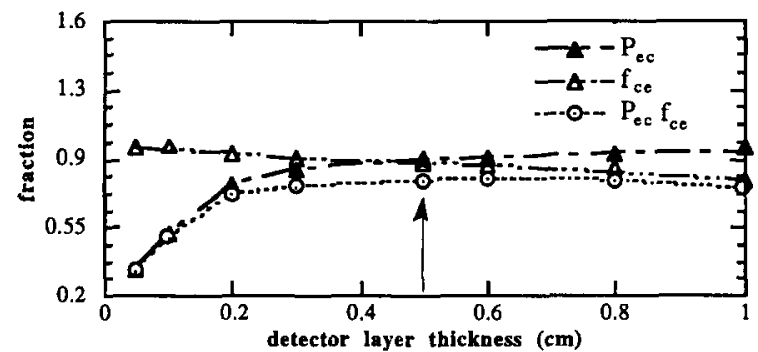

(c)

Fig. 2. Results from Monte Carlo simulations showing the fraction of single Compton scattering events followed by an escape of scattered gamma ray within a layer, $f_{c e}$, and the fraction of events in which recoll electron is captured in layer, $p_{\mathrm{ec}}$, as a function of layer thickness for (a) $150 \mathrm{keV}$, (b) 511 $\mathrm{keV}$, and (c) $1000 \mathrm{keV}$ normally incident gamma rays. 


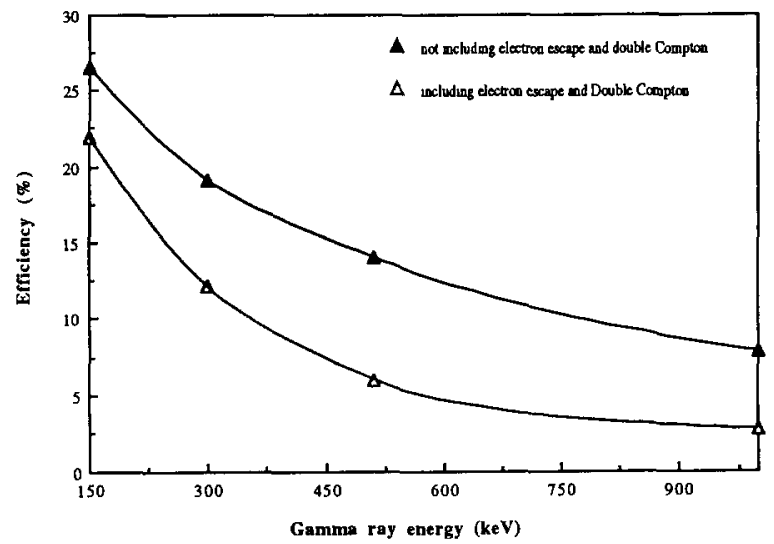

Fig. 3. Simulation predictıons of the intrinsic efficiency as a function of incident gamma ray energy for 20 layers of $1 \mathrm{~mm}$ thick Si surrounded by a $21 \mathrm{~cm}$ high Csl side counter. Note that the simulations which do not account for recoil electrom escape and multiple scatterngs overpredict the efficiency substantially at higher energies.

proposed design gave efficiencies of $2.5-22 \%$ over the range of $150-1000 \mathrm{keV}$ gamma rays. If electron escape and multiple scatterings were not included in our simulations, the efficiencies would have been incorrectly calculated to be $8-26 \%$. These additional events produce unwanted signals which will not reproduce the source position correctly. Because the range of the recoil electrons becomes larger than the layer thickness at high energies, the efficiency $(2.5 \%)$ and signal-tonoise ratio of the proposed design is low for $1000 \mathrm{keV}$ gamma rays. However, for a $4 \mathrm{~mm}$ (as opposed to 1 $\mathrm{mm}$ ) layer thickness, an efficiency of $8.8 \%$ (15\% if multiple scatterings and electron escape were not included) was calculated for $1000 \mathrm{keV}$ gamma rays. Note that these efficiencies are orders of magnitude greater than what has been achieved from electronic collimation using only two detector arrays $[5,6]$.

\subsection{Analytıc prediction of angular resolution for multiple scattering}

The accuracy of the multiple Compton method depends on the accuracy with which the cone parameters are determined from the measurements of the position and energy deposition values in the detector layers. In general, the measurement uncertainties cause a spread about the true location of the point source and are described by the point source response function (PSRF). The total contribution to the PSRF is calculated from

$\sigma_{\theta_{\text {total }}}=\left(\sigma_{\theta_{\mathrm{E}}}^{2}+\sigma_{\theta_{\mathrm{P}}}^{2}\right)^{05}$,

where $\sigma_{\theta_{\mathrm{E}}}$ and $\sigma_{\theta_{\mathrm{p}}}$ are the contributions from the energy and position resolutions of the detectors.

\subsubsection{Energy resolution contribution to angular resolu-} tion

The contribution of PSRF by the energy resolutions of the Si detectors is given by [7]

$$
\begin{aligned}
\left(\sigma_{\Delta E}^{\mathrm{FWHM}}\right)^{2}= & 2.35^{2} F W \Delta E+\left(\sigma_{\Delta E_{\mathrm{A}}}^{\mathrm{FWHM}}\right)_{\text {noise }}^{2} \\
& +\left(\sigma_{\Delta E_{\mathrm{A}}}^{\mathrm{FWHM}}\right)_{\text {leak dge }}^{2}
\end{aligned}
$$

where $F$ is the Fano factor, $W$ is the average ionization energy, and $\Delta E$ is the deposited energy in the detector layer by the electron, $\left(\sigma_{\Delta E_{\mathrm{A}}}^{\mathrm{FWM}}\right)_{\text {nore }}^{2}$ is the equivalent noise added by the preamplifier-amplifier combination and is expressed in terms of the equivalent noise charge (ENC), $\left(\sigma_{\Delta E_{A}}^{\mathrm{FWM}}\right)_{\text {leakage }}^{2}$ is the equivalent spread due to detector leakage current and charge collection variations in the detector. The noise term plays an important role for determination of the energy resolution of the Si strip detectors. One of the most important noise sources is the capacitance of the strips and the connections [8]. Consequently, depending on the choice of preamplifier-amplifier combination and the length of the strips, the energy resolutions of the strip detectors can vary significantly. In our calculations, the noise term was varied between $1-10 \mathrm{keV}$ and the values for $F$ and $W$ were chosen to be: $F=0.17$ [2], $W^{\mathrm{S}_{1}}=3.62 \mathrm{cV}$ [7].

The contributions from the energy resolution of the detectors transform into an uncertainty in Compton scatter angle $\theta$ (or the cone's vertex angle). Referring to Fig. 4, since the direction of the incident gamma ray is determined from the first two Compton interactions in the silicon layers, the contributions of the energy resolutions of these silicon detector layers to the angular resolution of the camera are calculated using the Compton scattering equation:

$\cos \theta_{1}=\left[1-\frac{m_{0} c^{2} \Delta E_{\mathrm{A}}}{\left(E_{\gamma 1}+\Delta E_{\mathrm{A}}\right) E_{\gamma 1}}\right]$,

where $\Delta E_{\mathrm{A}}$ is the energy deposited by the recoil electron in layer A, and $E_{\gamma t}$ is the energy of the scattered gamma ray after the first interaction. For convenience, we assume that the first interaction takes place in layer

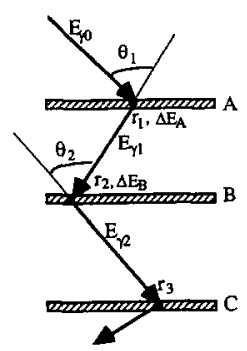

Fig. 4. The first three interaction positions and energy depositions in layers $\mathrm{A}, \mathrm{B}$ and $\mathrm{C}$. 
A, the second in layer B, and so forth. In practice, the order is determined using a sequence reconstruction algorithm described in ref. [3]. The uncertainty in the Compton scattering angle $\theta_{1}$ can be calculated by error propagation. Differentiation of Eq. (3) with respect to $\Delta E_{\mathrm{A}}$ and $E_{\gamma 1}$ gives the uncertainty in the Compton scattering angle (or cone half angle) according to the relation:

$$
\begin{aligned}
\sigma_{\theta_{\Sigma}}^{\text {multuple }}= & {\left[\left(\frac{\partial \theta_{1}}{\partial \cos \theta_{1}}\right)^{2}\left(\frac{\partial \cos \theta_{1}}{\partial \Delta E_{\mathrm{A}}}\right)^{2} \sigma_{\Delta E_{\mathrm{A}}}^{2}\right.} \\
& \left.+\left(\frac{\partial \theta_{1}}{\partial \cos \theta_{1}}\right)^{2}\left(\frac{\partial \cos \theta_{1}}{\partial \Delta E_{\gamma 1}}\right)^{2} \sigma_{E_{\gamma 1}}^{2}\right]^{1 / 2},
\end{aligned}
$$

where $\sigma_{E_{\gamma 1}}$ is the uncertainty in the scattered gamma ray energy $E_{\gamma 1}$. The energy of the scattered gamma ray $E_{\gamma 1}$ is calculated using the relation:

$$
\begin{aligned}
E_{\gamma 1}= & \left\{\Delta E_{\mathrm{B}}\left(1-\cos \theta_{2}\right)+\left[\left(1-\cos \theta_{2}\right)^{2} \Delta E_{\mathrm{B}}^{2}\right.\right. \\
& \left.\left.+4\left(1-\cos \theta_{2}\right) m_{0} c^{2} \Delta E_{\mathrm{B}}\right]^{1 / 2} / 2\left(1-\cos \theta_{2}\right)\right\}^{-1},
\end{aligned}
$$

where $\Delta E_{\mathrm{B}}$ is the energy deposited by the electron in layer $\mathrm{B}$ and $\theta_{2}$ is the angle of second Compton scattering which is calculated from the first three interaction locations. Then, $\sigma_{E_{y \mid}}$ can be calculated by error propagation by applying the relation:

$\sigma_{E_{\gamma 1}}=\left[\left(\frac{\partial E_{\gamma 1}}{\partial \theta_{2}}\right)^{2} \sigma_{\theta_{2}}^{2}+\left(\frac{\partial E_{\gamma 1}}{\Delta E_{\mathrm{B}}}\right)^{2} \sigma_{\Delta E_{\mathrm{B}}}^{2}\right]^{1 / 2}$

to Eq. (5). Our interest at this point is to explore the role of energy resolution on angular resolution so we shall assume the $\sigma_{\theta_{2}}$ term is comparatively negligible. For our proposed design, this is a reasonable approximation. Fig. 5 shows a surface plot of $\sigma_{\theta_{E}}^{\text {multiple }}$ using Eqs. (2), (4), (5) and (6) as a function of the Compton scattering angles $\theta_{1}$ and $\theta_{2}$ for $662 \mathrm{keV}$ incident gamma rays. We have assumed $5 \mathrm{keV}$ for the noise term in Eq. (2) and let $\sigma_{\theta_{2}}$ be negligibly small. As may be seen, the angular resolution is excellent for $\theta_{1} \approx 30-90^{\circ}$ and $\theta_{2}$ not small, and has a value of less than $2^{\circ}$. Note that with three or more interactions, the incident gamma ray does not need to deposit its full energy in the camera to reconstruct the incident direction and energy.

\subsubsection{Position resolution contribution to angular resolu- tion}

The second contribution to the PSRF is due to the position resolution of the strip detectors. Silicon strip detectors have excellent position resolutions, reaching down to a $4 \mu \mathrm{m}$ resolution using very thin strips [9]. In

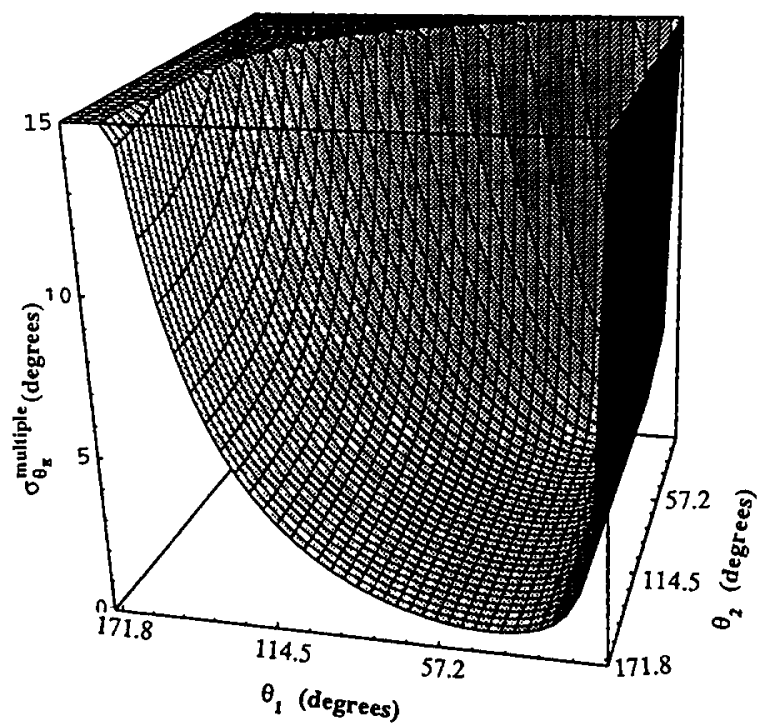

Fig. 5. A surface plot of $\sigma_{\theta_{t}}^{\text {multiple }}$ as a function of Compton scattering angles $\theta_{1}$ and $\theta_{2}$ for $662 \mathrm{keV}$ incident gamma rays assuming $5 \mathrm{keV}$ for the noise term.

our calculation, we assumed that the position resolution of the strip detector is equal to the pitch of the strips $(1 \mathrm{~mm})$. This leads to a value for $\sigma_{\mathrm{P}}$ of $1.5^{\circ}$ FWHM for the proposed design using Monte Carlo simulated data (see section 3.3 for the details). The total angular resolution $\sigma_{\theta_{1}}$ is then calculated from Eq. (1) using $\sigma_{\theta_{\mathrm{P}}}$ and $\sigma_{\theta_{\Gamma}}^{\text {multiple }}\left(\mathrm{E}_{\gamma 0}, \theta_{1}, \theta_{2}\right)$ for multiple scatterings and yields a value of $\sim 2.5^{\circ}$ over the scattering ranges mentioned in section 3.2.1.

\subsection{Monte Carlo simulation of the total angular resolu- tion}

The contributions of the energy and position resolutions of the detectors to the total angular resolution of the camera can be independently evaluated using the exact Monte Carlo data. For example, the broadening of the PSRF due to only the uncertainty in the energy $\Delta E$ deposited in a $S i$ detector can be determined using positions at their "true" values, but allowing a spread of values for $\Delta E$. The angular resolution due to the energy resolution of the detector can then be determined using these data points. Similarly exact values of $\Delta E$ can be used with a spread in interaction positions to find the position resolution contribution.

Monte Carlo simulations using a pencil beam source incident normally onto the camera were used to derive the angular resolution data. The actual values for the position of the interactions in the detectors and the energy deposited, $\Delta E$, were taken from the Monte Carlo simulations. The errors due to the energy resolution of the strip detectors for the first interaction only 
were simulated in the Monte Carlo runs by assuming that the energy deposition readout from the detector for a given Monte Carlo event is given by sampling from a Gaussian distribution centered on the simulated energy deposition $\Delta E_{\mathrm{A}}$ with FWHM of $\sigma_{\Delta E_{\mathrm{A}}}^{\mathrm{FWHM}}$ given by Eq. (2). For this simulation, we are assuming that the incident energy is known, thus only the first energy deposition contributes to the angular resolution. Then the backprojected cones for a given set of data were calculated by using the exact interaction positions and the modified energy deposition values. Similarly, the effects of the errors due to the interaction positions were calculated by using the position resolution of the Si strip detectors (assumed to be $1 \mathrm{~mm}$ FWHM in $x$ and $y$ and $z$ coordinates). A Gaussian distribution centered at the $x, y$, and $z$ coordinate of the interactions with FWHM of $1 \mathrm{~mm}$ was sampled to simulate the measurement. The backprojected cones were then calculated by using the modified interaction positions and the exact energy deposition values for each Monte Carlo event. The PSRF was calculated by tabulating the distributions of the angular difference between the true direction and the closest angle in the backprojected cones obtained from the modified energy and interaction positions. The total contribution to the PSRF was then calculated using Eq. (1). This approach should give a better estimate of the absolute resolution of the system than analyzing images after using an image reconstruction technique.

We have simulated 10000 gamma rays to determine the PSRF of the camera and calculated the angular difference between the true source direction and the backprojected cone for each event. Fig. 6 shows the spectrum of the number of different sequences of events from a Monte Carlo simulation of the proposed multiple Compton scatter imaging camera. Note that the most probable sequence for $662 \mathrm{keV}$ is the nonpro-

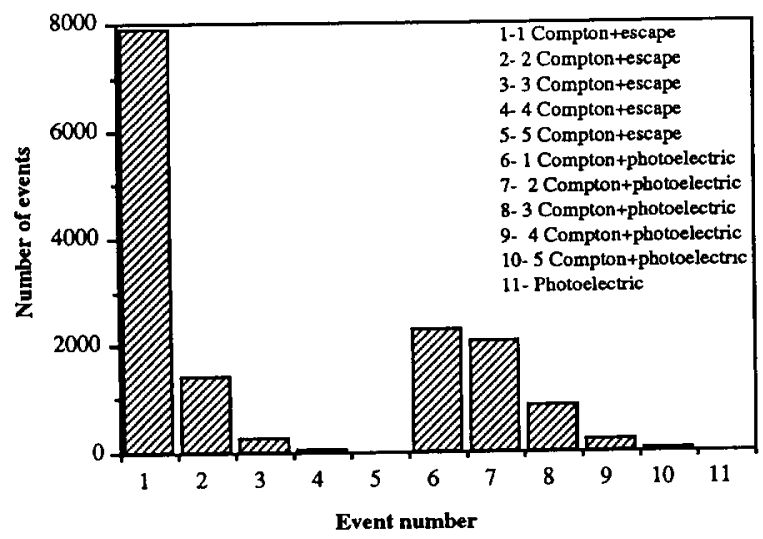

Fig. 6. A spectrum of number of events from a Monte Carlo simulation in the multiple Compton scatter gamma ray imaging camera ( $662 \mathrm{keV}, 50000$ photon history).

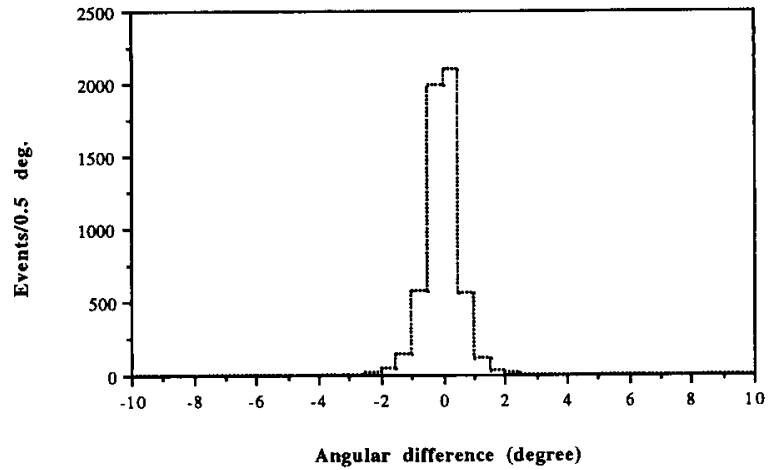

(a)

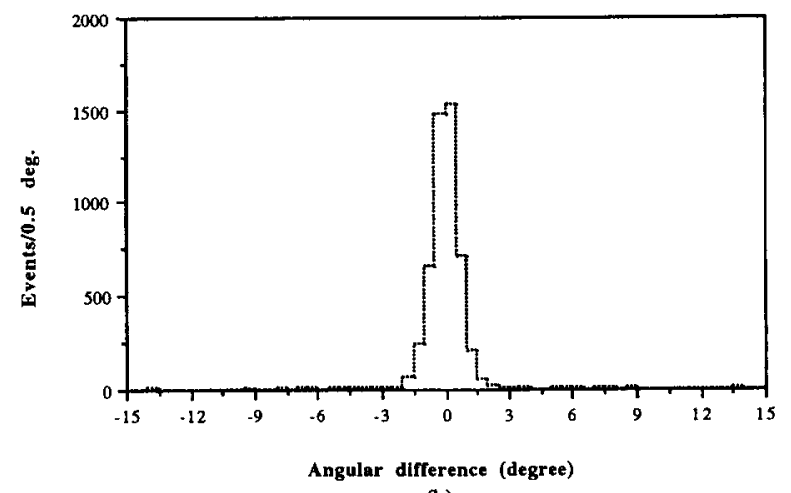

(b)

Fig. 7. Distributions of angular difference between the measured and true direction of the $511 \mathrm{keV}$ incident gamma rays due to (a) the energy resolution (assuming $1 \mathrm{keV}$ noise), (b) the position resolution of the Si strip detectors (assuming 1 $\mathrm{mm}$ position resolution).

ductive single Compton scattering followed by an escape. The PSRF calculations did not include any spreads due to an image reconstruction algorithm. In reality, the image reconstruction algorithm will also introduce extra spread around the source. Figs. $7 \mathrm{a}-7 \mathrm{~b}$ show the distributions of angular difference between the measured and the true direction when the energy and the position resolution of the strip detectors are applied separately for $511 \mathrm{keV}$ gamma rays, giving a total angular resolution $\left(\sigma_{\theta_{\text {total }}}\right)$ of approximately $2.8^{\circ}$, $4.5^{\circ}$, and $7.5^{\circ}$ (FWHM) assuming 1,5 , and $10 \mathrm{keV}$ noise terms respectively. The noise range we have chosen to investigate, $1-10 \mathrm{keV}$, represents realistic values [9]. Table 1 summarizes the total angular resolution of the camera for 150,511 , and $1000 \mathrm{keV}$ incoming gamma ray cnergies. The results showed that the contribution of the position resolution component $\left(\sigma_{\theta_{\mathrm{P}}}\right)$ to total angular resolution (1.5 $5^{\circ}$ FWHM assuming 1 $\mathrm{mm}$ position resolution) is much less than the energy resolution component $\left(\sigma_{\theta_{\mathrm{E}}}\right)$ over the energy range 150$511 \mathrm{keV}$. Note that the angular resolution improves with increasing energy, but is strongly dependent on 
Table 1

Summary of the angular resolution components of the multiple Compton scatter camera from Monte Carlo simulations assuming the incident gamma ray energy is known

\begin{tabular}{lcccc}
\hline$E_{\mathrm{g}}[\mathrm{keV}]$ & $\sigma_{\theta \mathrm{q}}[\mathrm{deg}]$ & $\sigma_{\theta_{I}}[\mathrm{deg}]$ & $\begin{array}{c}\text { Noise level } \\
{[\mathrm{keV}]}\end{array}$ & $\sigma_{\theta_{\mathrm{tat} a \mathrm{l}}}[\mathrm{deg}]$ \\
\hline 150 & 1.5 & 5.65 & 1 & 5.85 \\
& & 12.7 & 3 & 13.0 \\
511 & \multirow{2}{*}{1.5} & 2.00 & 1 & 2.50 \\
& & 4.24 & 5 & 4.50 \\
& & 7.14 & 10 & 7.50 \\
1000 & 1.5 & 0.90 & 1 & 1.75 \\
& & 2.00 & 5 & 2.50 \\
& & 3.16 & 10 & 3.50 \\
\hline
\end{tabular}

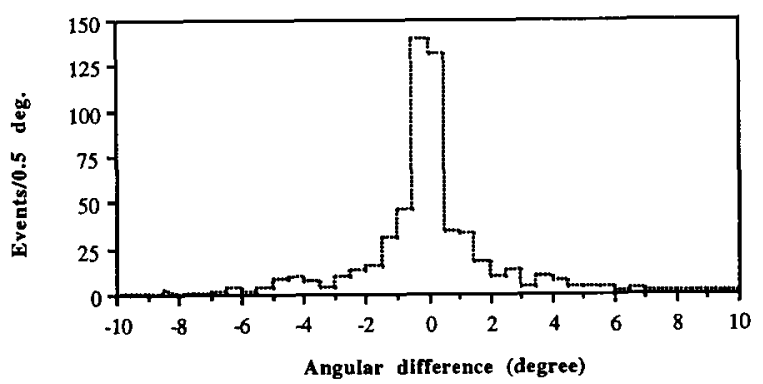

(a)

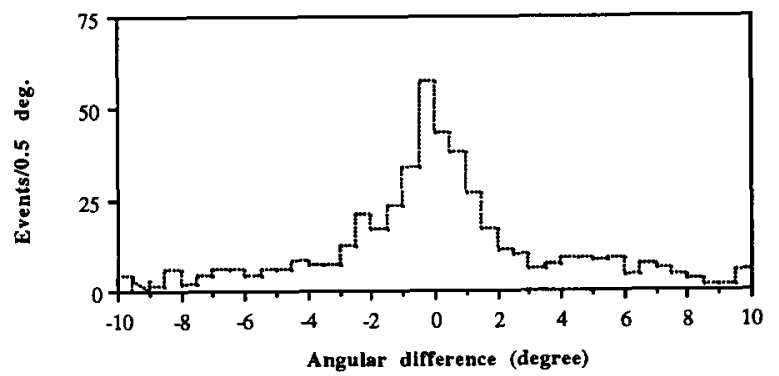

(b)

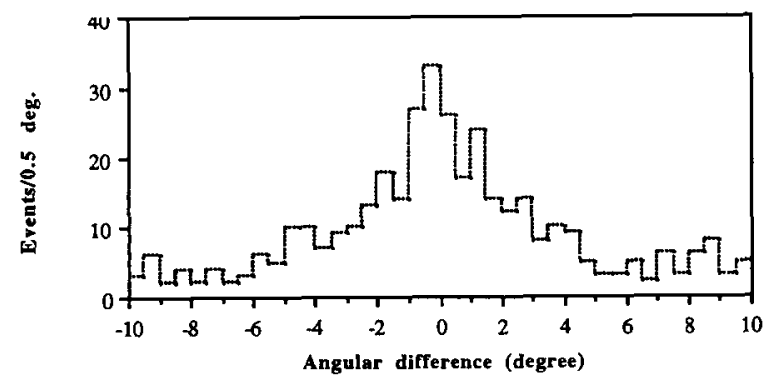

(c)

Fig. 8. Distributions of angular difference between the measured and true direction of the $662 \mathrm{keV}$ incident gamma rays due to the energy resolution of the strip detectors for the first two Compton interaction assuming (a) $1 \mathrm{keV}$ noise, (b) $5 \mathrm{keV}$ noise, and (c) $10 \mathrm{keV}$ noise. the energy resolution of the strip detectors, which in turn depends strongly on the noise source. The most important noise sources are the capacitance of the strips and connections (preamplifier and amplifier). Consequently, depending on the choice of electronics and layer specifications, the energy resolutions of the strip detectors can vary significantly. As seen from Table 1 , the energy resolution significantly improves when the noise level is decreased from 10 to $1 \mathrm{kcV}$ at all energies. Moreover, the angular resolution of the camera can be improved by excluding low and high angle scatterings at the expense of efficiency, although we have not investigated this trade-off.

In the above angular resolution calculations, we assumed the errors were due to the energy resolution of the strip detector for the first Compton interaction only $\left(\sigma_{\Delta E_{\mathrm{A}}}^{\mathrm{FWHM}}\right)$, assuming the incident gamma ray energy is known. However, as discussed in section 3.2.1, for a multiple Compton scatter camera, the direction of the incident gamma ray is determined from the first two Compton interactions in the silicon layers. Consequently, it is necessary to include the errors due to the energy resolutions of the strip detectors for the first two Compton interactions $\left(\sigma_{\Delta E_{\mathrm{A}}}^{\mathrm{HWHM}}, \sigma_{\Delta E_{\mathrm{B}}}^{\mathrm{FWHM}}\right)$. We simulated these errors assuming that the incident gamma ray energy is not known. Figs. $8 \mathrm{a}-8 \mathrm{c}$ show the distributions of angular difference between measured and true direction when energy resolutions of the first two strip detectors are applied for $662 \mathrm{keV}$ incident gamma rays, giving angular resolutions of approximately 1.7, 3, and $4.5^{\circ}$ (FWHM) assuming 1,5 , and $10 \mathrm{keV}$ noise respectively. The angular resolution assuming $5 \mathrm{keV}\left(3^{\circ}\right.$ FWHM) was estimated to be slightly worse than the analytically determined value $\left(\approx 2^{\circ}\right)$ for the average range of $\theta_{1} \approx 30-90^{\circ}$ and $\theta_{2}$ not small because we have not excluded low and high angles in our simulations. Nevertheless, the reasonable agreement between the analytic and simulated results is encouraging.

\section{Summary and conclusions}

In this work, the optimum layer thickness of a Compton camera which utilizes multiple Compton scatterings was found to be less than $2 \mathrm{~mm}$ for $150-511$ $\mathrm{keV}$ incident gamma rays and approximately $5 \mathrm{~mm}$ for $1000 \mathrm{keV}$ gamma rays. These results suggest that the detector layer thickness should be thinner for low energy gamma rays than for high energy gamma rays. Based on this optimization, we also presented the efficiency and the angular resolution of a proposed multiple Compton scatter imaging camera which consists of $1 \mathrm{~mm}$ thick, 20 layer Si strip detectors surrounded with an annular CsI side counter. Monte Carlo simulations of the prototype design gave angular resolutions $2-7^{\circ}$ (FWHM) assuming a $1 \mathrm{keV}$ noise 
Table 2

Summary of Monte Carlo estimates of angular resolution and intrinsic efficiencies for a multiple Compton scatter imaging camera for 20 layers of $1 \mathrm{~mm}$ thick $S_{1}$ surrounded by a $21 \mathrm{~cm}$ high CsI side counter

\begin{tabular}{llccl}
\hline $\begin{array}{l}E_{\gamma 0} \\
{[\mathrm{keV}]}\end{array}$ & \multicolumn{2}{l}{ Angular resolution [deg] } & $\begin{array}{l}\text { Noise } \\
\text { level } \\
{[\mathrm{keV}]}\end{array}$ & $\begin{array}{l}\text { Efficiency } \\
{[\%]}\end{array}$ \\
\hline 150 & 5.85 & 7.15 & 1 & 22.0 \\
& 13.0 & 17.56 & 3 & \\
511 & 2.5 & 2.78 & 1 & 7.0 \\
& 4.5 & 5.12 & 5 & \\
& 7.5 & 8.87 & 10 & \\
1000 & 1.75 & 1.89 & 1 & 2.5 \\
& 2.5 & 2.91 & 5 & \\
& 3.5 & 5.22 & 10 & \\
\hline
\end{tabular}

source with intrinsic efficiencies of $2.5-22 \%$ over the energy range of $150-1000 \mathrm{keV}$. The final results are summarized in Table 2. Although the angular resolutions obtained are rather modest, the efficiency is very encouraging. This high efficiency suggests that the multiple Compton scatter camera may be particularly useful for imaging weak radiation sources, and minimizing image acquisition times.

\section{References}

[1] T. Kamea and H. Hanada, IEEE Trans. Nucl. Sci. NS-35 (1988) 352.

[2] J.T. Walton, Nucl. Instr. and Meth. 226 (1984) 1.

[3] N. Dogan, D. Wehe and G. Knoll, Nucl. Instr. and Meth. A 299 (1990) 501.

[4] A. Halbleib and A. Melhorn, Sandia National Laboratory, report SAND 84-0573 (1984).

[5] R.R. Brechner and M. Singh, IEEE Trans. Nucl. Scı. NS-35 (1988) 649.

[6] J.B. Martin, G.F. Knoll, D.K. Wehe, N. Dogan, V. Jordanov, N. Petrick and M. Singh, IEEE Trans. Nucl. Sci. NS-40 (1993) 972.

[7] G.F. Knoll, Radiation Detection and Measurement, 1st ed. (Wiley, 1979) pp. 481.

[8] V. Radeka, Nucl. Instr. and Meth. 226 (1984) 209.

[9] C.D. Wilburn, Micron Semiconductor, private communication, 1993. 